\title{
Variations géographiques et saisonnières de la qualité du fromage de Comté
}

\author{
par \\ C. MASSON*, P. ROUSSEAUX** et C. DECAEN* \\ Avec la collaboration de P. GIRAUDON, Ch. PERROT \\ et D. REINHARDT
}

Le Comté est un fromage traditionnel qui, fabriqué selon des techniques semi-artisanales, assure la majeure partie du revenu des éleveurs laitiers d'une région (la Franche-Comté) où la production laitière reste la spéculation agricole dominante compte tenu des conditions écologiques et socio-économiques avec des petites structures au niveau des expoitations agricoles et des coopératives laitières, communément appelées fruitières.

Le Comté constitue un aliment noble et son appellation d'origine lui assure un marché défini exigeant une qualité constante. La qualité de ce produit résulte :

- de la qualité de la matière première : le lait ;

- des techniques de fabrication et d'affinage mises en œuvre pour l'obtention de ce fromage.

Mais, bien que fabriqués dans une zone géographique limitée et selon des procédés technologiques assez immuables, la qualité des fromages de Comté subit des variations assez importantes. L'analyse en est complexe en raison des nombreuses composantes de cette qualité et des multiples facteurs agissant tant sur le lait qu'au niveau des techniques de fabrication et d'affinage. Cependant pour les producteurs, il est souhaitable de contrôler au mieux ces variations et d'alimenter le marché avec une production de bonne qualité homogène.

C'est pourquoi, devant l'absence d'étude systématique sur ce problème, nous avons entrepris une étude sur la qualité du fromage de Comté et les facteurs susceptibles d'influencer cette qualité. Après

\footnotetext{
* Laboratoire de la Chaire de Production Animale ENSSAA - 21000 Dijon.

** Station laitière expérimentale INRA - 39800 Poligny.
} 
avoir décrit les variations saisonnières et géographiques de la composition chimique des laits de quelques fruitières [5], nous avons voulu analyser les variations de la qualité du fromage en essayant de maîtriser l'influence de la technologie fromagère.

\section{MATERIEL - METHODE}

\section{Schéma expérimental}

Nous avons choisi trois fruitières de Franche-Comté en fonction de leur situation géographique :

- pour la zone de plaine et basse vallée (inférieur à $400 \mathrm{~m}$ ), la fruitière A ;

- pour la zone de plateau (400 à $800 \mathrm{~m}$ ), la fruitière $\mathrm{B}$;

- pour la zone de montagne (plus de $800 \mathrm{~m}$ ), la fruitière $\mathrm{C}$.

Cinq fabrications sont étudiées au cours de l'année aux périodes suivantes : novembre, janvier, mars, mai, septembre. En vue de contrôler l'effet des conditions de fabrication qui diffèrent sur certains points d'une fruitière à l'autre, des fromages sont fabriqués avec le lait des producteurs de chacun des châlets étudiés, d'une part dans la fruitière elle-même, et d'autre part dans la fromagerie expérimentale annexe de I'INRA à Aresches (39).

A chaque série, deux fromages sont fabriqués, à la fruitière d'Aresches portant référence : INRA I et INRA II. Un fromage du même jour, fabriqué dans la fruitière ayant livré le lait nécessaire aux fabrications précédentes, porte référence " Fruitière I " et enfin un fromage fabriqué dans la même fruitière, environ 1 semaine après, qui est appelé "Fruitière II ». De plus, afin d'éliminer des variations possibles dans la méthode d'affinage au niveau des fromages provenant des trois fruitières et de la fromagerie d'Aresches, ceux-ci sont affinés dans les mêmes locaux chez un affineur et à des conditions identiques de température et d'hygrométrie.

\section{Prélèvements}

A chaque fabrication, un échantillon de lait de mélange avant emprésurage est prélevé dans la cuve pour l'analyse chimique. Ce lait est écrémé partiellement pour obtenir un fromage ayant une teneur en matière grasse dans l'extrait sec conforme au décret sur l'appellation d'origine du Comté.

$\mathrm{Au}$ niveau des fromages, l'une des meules fabriquées à Aresches (INRA I) est utilisée pour les analyses chimiques ; trois sondes sont prélevées à différents stades d'affinage : $20 \mathrm{~h}, 3$ mois, 4 mois, 5 mois et 6 mois et ceci pour les cinq séries réparties sur l'année. 
Des sondes sont, en outre, prélevées sur les trois autres fromages : INRA II, fruitière I et fruitière II de chaque fromagerie (c'està-dire neuf au total) à 3 et 5 mois d'âge à raison d'une sonde par fromage.

A 6 mois d'âge, ces fromages sont coupés et un prélèvement est opéré. d'âge.

Les fromages sont pesés à $20 \mathrm{~h}, 3$ mois, 4 mois, 5 mois et 6 mois

\section{Analyses}

\section{Analyses chimiques :}

- sur le lait : les taux butyreux et azoté sont déterminés respectivement par la méthode Gerber et la méthode au Noir Amido ; la composition en acides gras de la matière grasse est réalisée par chromatographie en phase gazeuse d'esters butyliques et exprimée en acides gras courts $\left(\mathrm{C}_{4}-\mathrm{C}_{12}\right)$, en acides gras moyens $\left(\mathrm{C}_{12}-\mathrm{C}_{16}\right)$ et acides gras longs $\left(>\mathrm{C}_{16}\right)$;

- sur les fromages : toutes les analyses sont faites sur du fromage broyé. L'extrait sec du fromage est calculé après une dessiccation dans une étuve à $102^{\circ} \mathrm{C} \pm 1^{\circ} \mathrm{C}$ pendant $24 \mathrm{~h}$. La teneur en matière grasse du fromage est réalisée par la méthode de Van Gulik qui, bien que moins précise que la méthode officielle, suffit pour les comparaisons entre fruitières. La composition en acides gras estérifiés est analysée par chromatographie en phase gazeuse, après avoir séparé les acides gras libres des acides gras estérifiés. Nous avons effectué une mesure approximative de protéolyse en dosant la teneur apparente en protéines du fromage par la méthode au Noir Amido. L'indice de protéolyse utilisé représente la variation relative de la mesure Noir Amido faite sur le fromage affiné par rapport à la mesure faite sur fromage à $20 \mathrm{~h}$, les mesures étant corrigées en fonction de l'extrait sec du fromage.

\section{ANALYSE SENSORIELLE}

L'analyse des caractères organoleptiques est effectuée sur les neuf fromages mentionnés au sous-chapitre prélèvement ( ${ }_{3}$ INRA II, ${ }_{3}$ fruitière I, ${ }_{3}$ fruitière II) et à l'âge prévu.

Deux critères sont retenus : la texture de la pâte et le goût ; au $6^{\mathrm{e}}$ mois s'ajoute la notation de l'ouverture.

Les contraintes expérimentales (nombre de fromages, nombre de dégustateurs et la quantité de fromages disponibles) nous ont conduits à utiliser la méthode des blocs incomplets équilibrés. Ainsi le plan adopté nécessite douze dégustateurs, chacun d'eux jugeant trois échantillons au point de vue de la texture, trois échantillons différents du point de vue du goût, et enfin, trois échantillons dif- 
férents (uniquement au $6^{\mathrm{e}}$ mois d'affinage) pour l'appréciation de l'ouverture.

\section{RESULTATS}

\section{Composition chimique des laits et des fromages}

Les résultats des analyses chimiques faites tant sur le lait que sur les fromages issus de ces fabrications nous permettent de définir un produit moyen de base, le lait, et un produit moyen fini, le fromage à 6 mois d'affinage dont les caractéristiques figurent dans le tableau 1.

\section{TABLEAU 1}

Composition chimique moyenne du lait et du fromage à 6 mois (moyenne et écart-type)

\begin{tabular}{|c|c|c|}
\hline Critères analysés & Lait & Fromage à 6 mois \\
\hline Extrait sec (p. 100) & - & $65,0 \pm 0,5$ \\
\hline $\mathrm{pH}$ & - & 5,7 \\
\hline Matière grasse & (*) $31,9 \pm 1,5 \mathrm{~g} / \mathrm{kg}$ & $48,3 \pm 0,8 \mathrm{~g} / 100 \mathrm{~g} \mathrm{MS}$ \\
\hline $\begin{array}{l}\text { Acides gras estérifiés } \\
\begin{array}{l}\text { (p. } 100 \text { molaire }): \\
- \text { courts }\left(\mathrm{C}_{4}-\mathrm{C}_{12}\right) \\
- \text { moyens }\left(\mathrm{C}_{12}-\mathrm{C}_{16}\right) \\
\text { - longs }\left(\mathrm{C}_{18}\right)\end{array}\end{array}$ & $\begin{array}{l}23 \pm 2 \\
43 \pm 5 \\
34 \pm 4\end{array}$ & $\begin{array}{l}26 \pm 2 \\
43 \pm 3 \\
31 \pm 3\end{array}$ \\
\hline Matière azotée & $32,2 \pm 1,2 \mathrm{~g} / \mathrm{kg}$ & $(* *) 24,4 \pm 1,8 \mathrm{~g} / 100 \mathrm{~g} \mathrm{MS}$ \\
\hline Chlorures $(\mathrm{NaCl})$ & $1,6 \mathrm{~g} / \mathrm{kg}$ & $1,0 \mathrm{~g} / 100 \mathrm{~g} \mathrm{MS}$ \\
\hline
\end{tabular}

${ }^{*}$ ) Teneur en matières grasses dans la cuve après écrémage partiel.

(**) Teneur apparente en matière azotée par la méthode au noir amido (explication p. 33).

A. VARIATIONS GÉOgRAPHiQues

Composition chimique du lait

Pour la composition en acides gras, les teneurs en matières azotées et en chlorures, aucune différence significative $(P<0,05)$ n'a pu être mise en évidence entre les trois fruitières (voir tab. 2). 
TABLEAU 2

Composition chimique des laits : comparaison entre zones géographiques

\begin{tabular}{|c|c|c|c|c|c|}
\hline Fruitères & $\begin{array}{c}\text { A.G. } \\
\text { courts } \\
\text { p. } 100 \\
\text { molaire }\end{array}$ & $\begin{array}{l}\text { A.G. } \\
\text { moyens } \\
\text { p. } 100 \\
\text { molaire }\end{array}$ & $\begin{array}{c}\text { A.G. } \\
\text { longs } \\
\text { p. } 100 \\
\text { molaire }\end{array}$ & $\begin{array}{l}\text { M.A.T. } \\
\text { g/kg }\end{array}$ & $\begin{array}{l}\text { Chlorures } \\
\mathrm{g} \mathrm{NaCl} / \mathrm{kg}\end{array}$ \\
\hline A & 23,1 & 42,2 & 34,2 & 32,1 & 1,57 \\
\hline B & 22,9 & 43,5 & 32,9 & 32,4 & 1,57 \\
\hline C & 22,6 & 42,2 & 35,1 & 32,0 & 1,58 \\
\hline Signification & NS (1) & NS & NS & NS & NS \\
\hline
\end{tabular}

(1) Non significatif.

Composition chimique des fromages à 6 mois d'affinage

La composition chimique diffère peu d'une fruitière à l'autre (voir tab. 3) ; seules des différences statistiques $(P<0,05)$ apparaissent pour les critères pourcentage molaire en acides gras longs et teneur en chlorures. Les fromages de $\mathrm{C}$ contiennent moins d'acides gras courts et moyens et plus d'acides gras longs que ceux de A et B.

$T A B L E A U 3$

Composition chimique des fromages à 6 mois d'affinage : comparaison entre zones géographiques

\begin{tabular}{|c|c|c|c|c|c|c|}
\hline Fruitières & $\begin{array}{l}\text { E.S. } \\
\text { p. } 100\end{array}$ & $\mathrm{pH}$ & $\begin{array}{l}\text { A.G. } \\
\text { courts } \\
\text { p. } 100 \\
\text { molaire }\end{array}$ & $\begin{array}{l}\text { A.G. } \\
\text { moyens } \\
\text { p. } 100 \\
\text { molaire }\end{array}$ & $\begin{array}{l}\text { A.G. } \\
\text { longs } \\
\text { p. } 100 \\
\text { molaire }\end{array}$ & $\begin{array}{l}\text { Chlorures } \\
\mathrm{g} \mathrm{NaCl} / \mathrm{kg}\end{array}$ \\
\hline A & 65,1 & 5,7 & 27,1 & 42,6 & 30,0 & 0,93 \\
\hline B & 65,0 & 5,8 & 24,8 & 43,8 & 29,4 & 0,98 \\
\hline C & 64,9 & 5,7 & 24,3 & 41,9 & 32,3 & 1,01 \\
\hline Moy. & 65,0 & 5,7 & 25,4 & 42,8 & 30,6 & 0,97 \\
\hline Signification & NS & NS & NS & NS & $S^{*}(1)$ & $\mathrm{S}^{*}$ \\
\hline
\end{tabular}




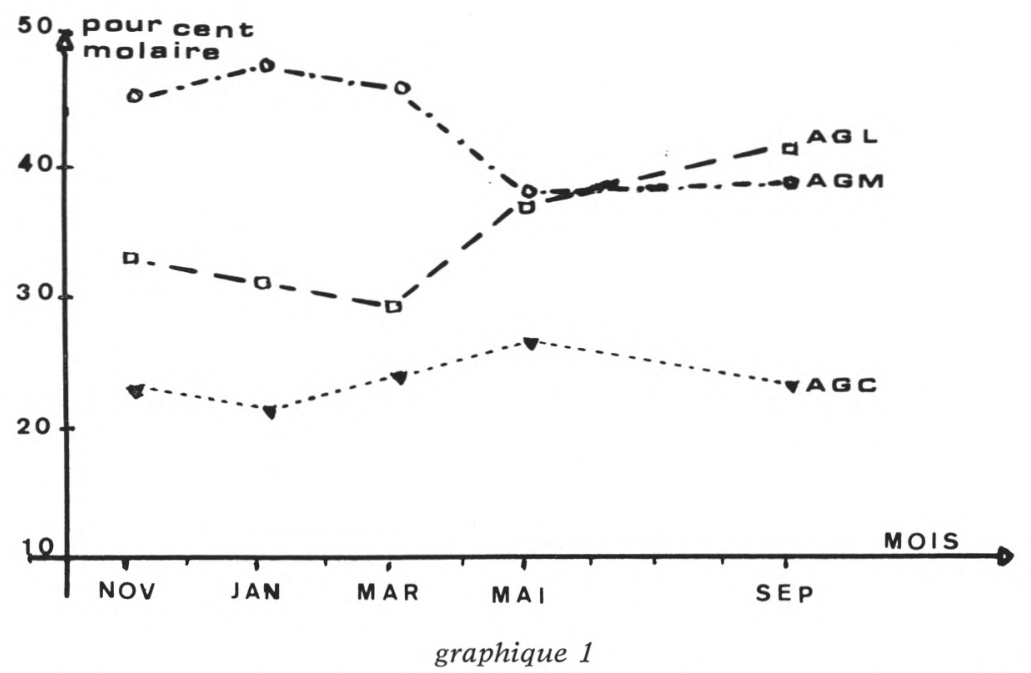

Composition moyenne en acides gras des lipides des laits de trois fruitières : acides gras longs (AGL) - - ; acides gras moyens (AGM) -...- ; acides gras courts (AGC)

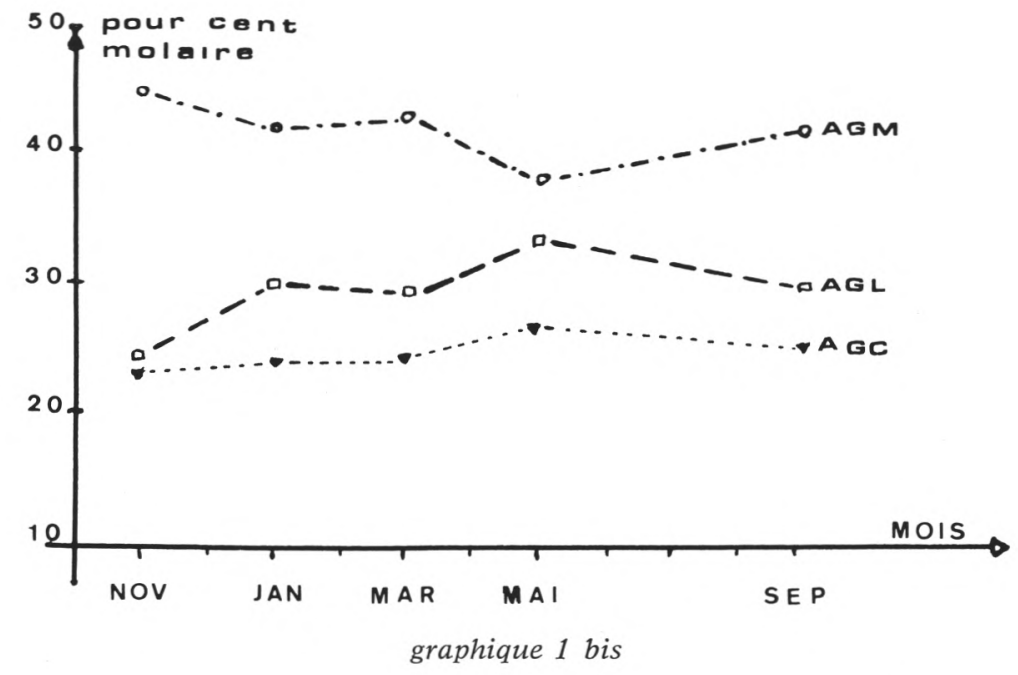

Composition moyenne en acides gras des lipides des fromages (Comté) de trois fruitières à 6 mois d'affinage. 


\section{B. VARIATIONS SAISONNIËRES}

\section{Lait}

La saison est un important facteur de variation de la composition du lait ; elle entraîne des changements hautement significatifs $(\mathrm{P}<0,01)$ pour la plupart des variables étudiées. De plus, l'évolution au cours de l'année est sensiblement la même pour les trois fruitières.

La composition en acides gras des matières grasses du lait évolue de façon considérable en fonction de la saison : les lipides du lait de la période de pâturage (mai-septembre) sont, par rapport à ceux du lait de la période de stabulation (novembre-janvier-mars), plus riches en acides gras courts $(24,4$ contre 21,8 p. 100), plus pauvres en acides gras moyens $(37,5$ contre 46,8 p. 100) et plus riches en acides gras longs $(38,3$ contre 31,2 p. 100$)$.

La teneur en matière azotée présente un maximum en novembre $(33,2 \mathrm{~g} / \mathrm{kg})$; elle accuse une diminution très nette en hiver $(31,1 \mathrm{~g} / \mathrm{kg}$ en janvier et $30,9 \mathrm{~g} / \mathrm{kg}$ en mars) et après une remontée en mai $(32 \mathrm{~g} / \mathrm{kg})$, elle atteint un deuxième maximum en septembre. La teneur en chlorures $(1,57 \mathrm{~g} \mathrm{NaCl} / \mathrm{kg})$ est la seule variable qui est stable au cours de l'année.

\section{Fromage}

La composition chimique des fromages varie de façon hautement significative en fonction de la saison pour l'ensemble des fruitières. De plus, l'interaction saison - fruitière est hautement significative pour les critères extrait $\mathrm{sec}$, matières grasses et matières azotées, c'est-à-dire que, selon la fruitière, l'évolution de ces critères en fonction de la saison n'est pas la même.

L'extrait sec moyen des fromages des trois fruitières à 6 mois d'affinage (graphique 2), minimum pour les fromages fabriqués en janvier (64,4 p. 100) est inférieur à ceux de septembre $(64,8$ p. 100) et mars $(64,9$ p. 100$)$ et nettement plus faible que ceux de novembre $(65,2$ p. 100$)$ et mai $(65,5$ p. 100$)$. Cette évolution diffère sensiblement selon les fruitières; le minimum est soit en janvier pour $B$ et $C$ soit en septembre pour $A$, mais le maximum est en mai pour deux fruitières $\mathrm{A}$ et $\mathrm{B}$ et en novembre pour la troisième $\mathrm{C}$.

La composition en acides gras des lipides du fromage à 6 mois d'affinage varie très peu d'un mois à l'autre.

Seules des différences significatives apparaissent pour les pourcentages molaires en acides gras moyens et en acides gras longs (graphique 1 bis). Ainsi les pourcentages molaires d'acides gras moyens des fromages de mai $(38,9)$ sont inférieurs à ceux de janvier $(42,8)$, septembre $(42,8)$, mars $(43,5)$ et novembre $(45,8)$ par contre, pour les acides gras longs, ce sont les fromages de mai $(34,1)$ qui sont plus élevés que ceux de janvier $(30,5)$, septembre $(30,5)$, mars (30) et novembre $(27,4)$. 
Les écarts entre les teneurs en chlorures sont très importants. Pour les trois fruitières, le minimum se situe en novembre $(0,67 \mathrm{~g}$ $\mathrm{NaCl}$ p. $100 \mathrm{MS})$ et le maximum en mars (0,93 g NaCl p. $100 \mathrm{MS})$; seules des différences entre fruitières apparaissent pour les mois intermédiaires.

En définitive la composition chimique des laits et des fromages à 6 mois d'affinage varie beaucoup plus en fonction de la saison que de la zone géographique comme en témoignent les écarts exprimés en p. 100 de la teneur la plus faible pour les différents critères figurant dans le tableau 4.

\section{TABLEAU 4}

Ecart de pourcentage molaire en p. 100 de la teneur la plus faible, des différents types d'acides gras du lait et du fromage.

Ecart pourcentage molaire

\begin{tabular}{l|c|c|c}
\hline \multicolumn{1}{c|}{ Variations de } & $\begin{array}{c}\text { Acides gras } \\
\text { courts }\end{array}$ & $\begin{array}{c}\text { Acides gras } \\
\text { moyens }\end{array}$ & $\begin{array}{c}\text { Acides gras } \\
\text { longs }\end{array}$ \\
\hline & & & \\
Caractère géographique : & 2,2 & 3,1 & 6,7 \\
- lait & 11,5 & 4,5 & 9,9 \\
- fromage & 11,9 & 30 & 22,7 \\
Caractère saisonnier : & 10,7 & 16 & 30,8 \\
- lait & & & \\
\hline
\end{tabular}

\section{VARIATIONS DE LA COMPOSITION CHIMIQUE AU COURS DE L'AFFINAGE}

Les sondes prélevées à 20 h, 3 mois, 4 mois, 5 mois et 6 mois au niveau des trois fruitières nous permettent de suivre l'évolution de la composition chimique des fromages au cours de l'affinage (graphiques 2 et 3 ).

Dans l'ensemble, les différences de composition chimique entre les différents stades d'affinage sont hautement significatives quelle que soit la fruitière. Quant à l'interaction mois-stade d'affinage, elle est, dans la plupart des cas, significative.

L'extrait sec, minimum à $20 \mathrm{~h}$ (63), évolue régulièrement jusqu'au $3^{\mathrm{e}}$ mois $(64,1)$ et $4^{\mathrm{e}}$ mois $(64,2)$ puis augmente de façon hautement significative au $5^{\mathrm{e}}$ mois $(65,1)$ pour se stabiliser au $6^{\mathrm{e}}$ mois $(65)$. Cependant cette évolution diffère selon le mois de fabrication. Ainsi, en 

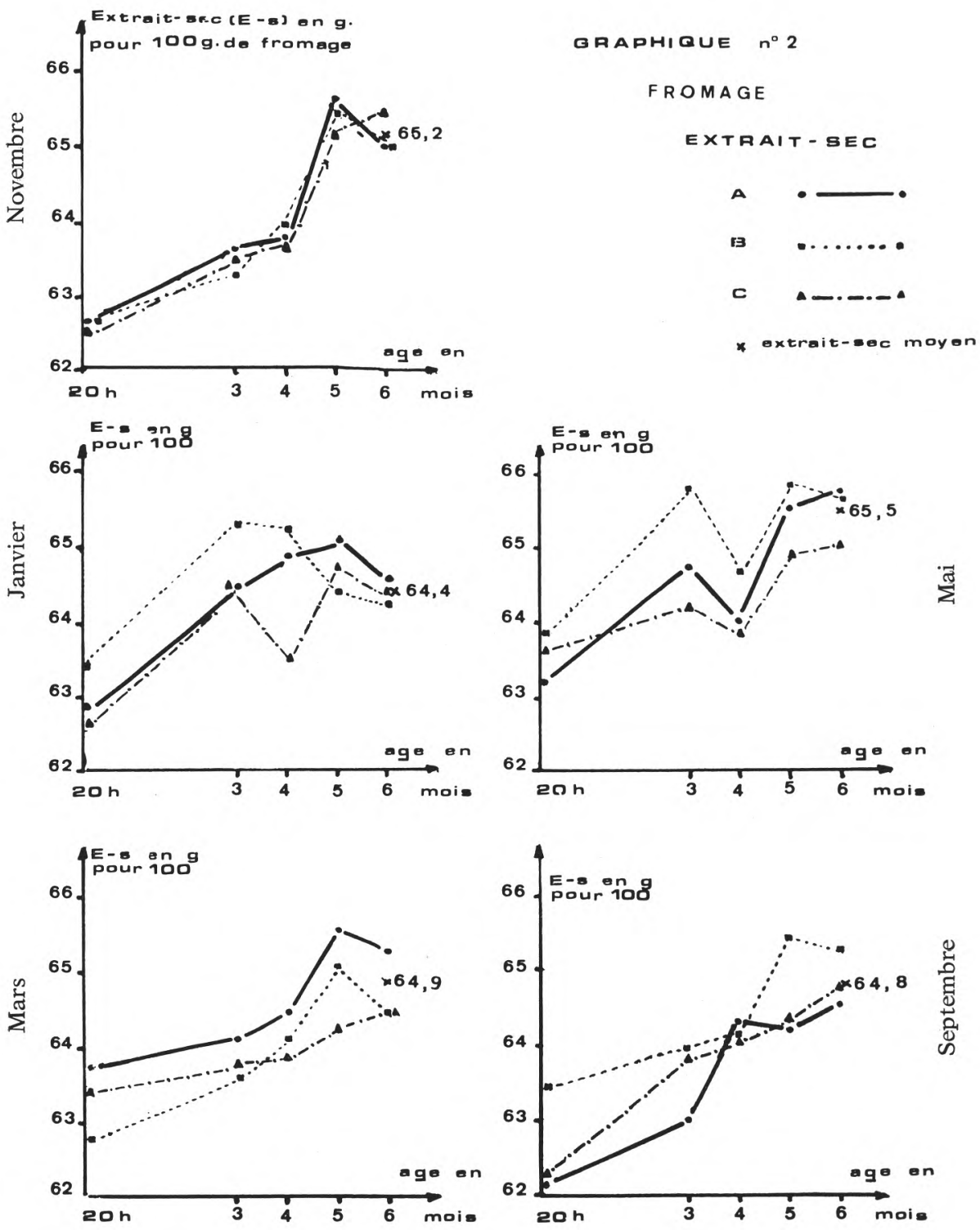

graphique 2 

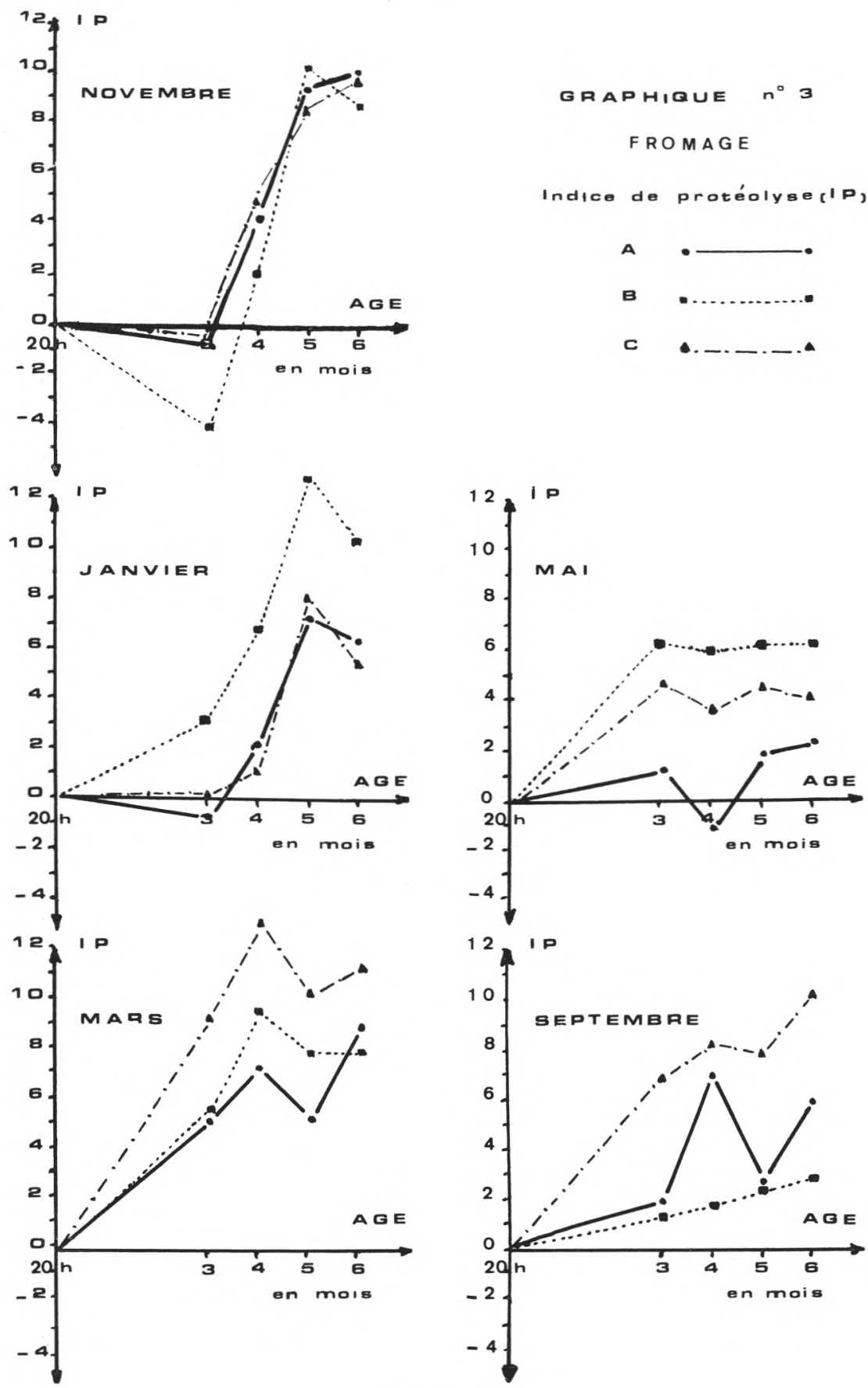

Indice de protéolyseclp
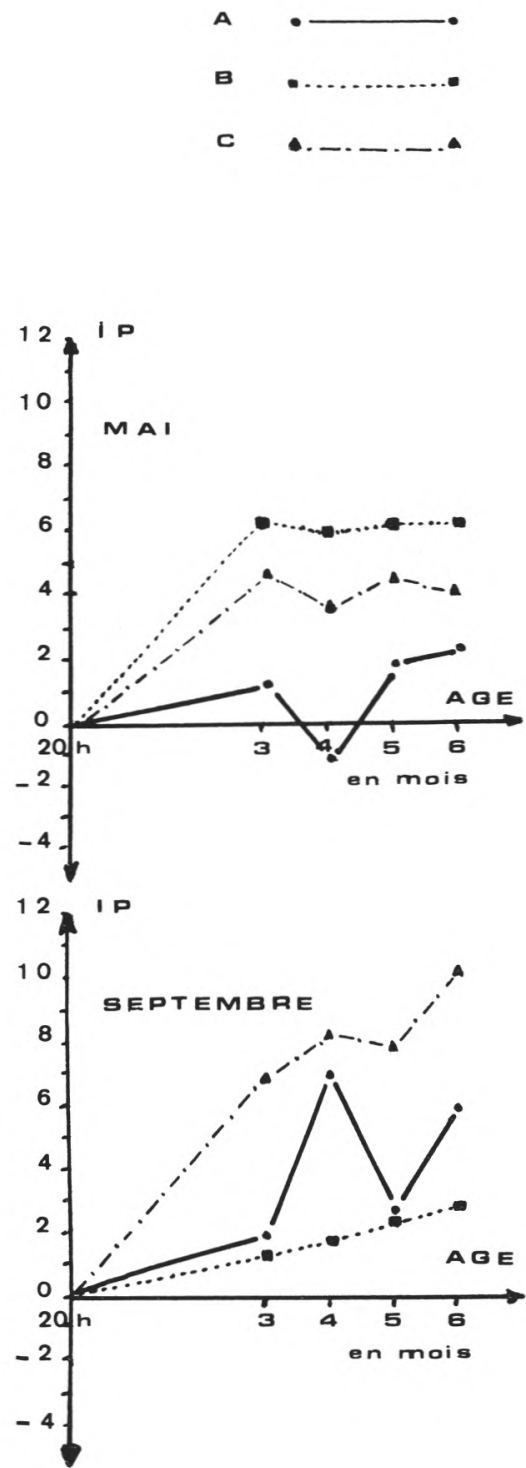

graphique 3 
janvier, seul l'extrait sec à $20 \mathrm{~h}$ (63) diffère des extraits secs du $6^{\mathrm{e}}$ mois $(64,4)$, du $4^{\mathrm{e}}$ mois $(64,5)$, du $3^{\mathrm{e}}$ mois $(64,8)$ et du $5^{\mathrm{e}}$ mois $(64,9)$.

Le $\mathrm{pH}$, minimum à $20 \mathrm{~h}(5,23)$, augmente brutalement au $3^{\mathrm{e}}$ mois $(5,60)$ pour se stabiliser ensuite ; les valeurs sont respectivement de $5,67,5,74$ et 5,74 pour les $4^{\mathrm{e}}, 5^{\mathrm{e}}$ et $6^{\mathrm{e}}$ mois.

La composition en acides gras des lipides des fromages est peu modifiée et peu caractéristique d'un stade d'affinage donné.

Au cours de l'affinage, l'indice de protéolyse augmente considérablement du stade $20 \mathrm{~h}$ au stade 5 mois pour se stabiliser au $6^{\mathrm{e}}$ mois (graphique 3). Cette évolution diffère selon le mois de fabrication ; ainsi en mai l'indice de protéolyse semble plus faible et diffère peu entre le $3^{\mathrm{e}}$ et le $6^{\mathrm{e}}$ mois ; en septembre, il en est sensiblement de même ; pour les mois de novembre, janvier et mars, l'évolution importante de l'indice de protéolyse se situe entre le $3^{\mathrm{e}}$ et le $5^{\mathrm{e}}$ mois.

La variation entre les teneurs extrêmes en chlorures est très importante, $0,30 \mathrm{~g} \mathrm{NaCl}$ p. $100 \mathrm{MS}$ à $20 \mathrm{~h}$ et $1 \mathrm{~g} \mathrm{NaCl}$ p. $100 \mathrm{MS}$ au $5^{\text {e }}$ mois [6]. Elle est due aux fréquents apports de sel sur les fromages qui sont frottés lorsque celui-ci est plus ou moins fondu.

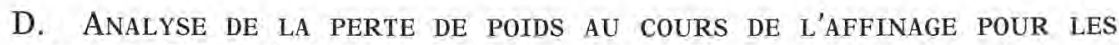
FROMAGES INRA I ET FRUITIÈRE I

La perte de poids observée entre $20 \mathrm{~h}$ et 4 mois d'affinage est représentative de la perte globale ; c'est pourquoi nous avons retenu l'âge de 4 mois pour mesurer la perte et calculer les corrélations apparentes entre la perte en poids et les autres variables mesurées. Ce choix est également justifié, nous semble-t-il, par le marché actuel du Comté qui ne conduit pas la plupart du temps à une conservation plus longue dans les caves.

\section{Variations saisonnières et géographiques}

L'analyse de variance montre qu'il n'y a pas de différence significative entre les pertes de poids des fromages INRA I ou fruitière I. De la même façon, l'effet zone géographique n'est pas significatif ; par contre l'effet saison l'est $(P<0,01)$; les interactions entre facteurs ne sont pas significatives. Dans l'ensemble la perte de poids à 4 mois la plus faible est observée pour les fromages fabriqués en mars (de 5,2 à 6,1 p. 100) sauf pour le fromage "Fruitière I " de B, en janvier, et, la plus forte en novembre de 8,8 à 10,4 p. 100 (graphique 4).

2. Corrélations entre la perte de poids et les autres variables mesurées

a) Format des fromages

Le format et la perte observée à 4 mois ne sont pas significativement correlés. L'idée selon laquelle les fromages les plus gros 


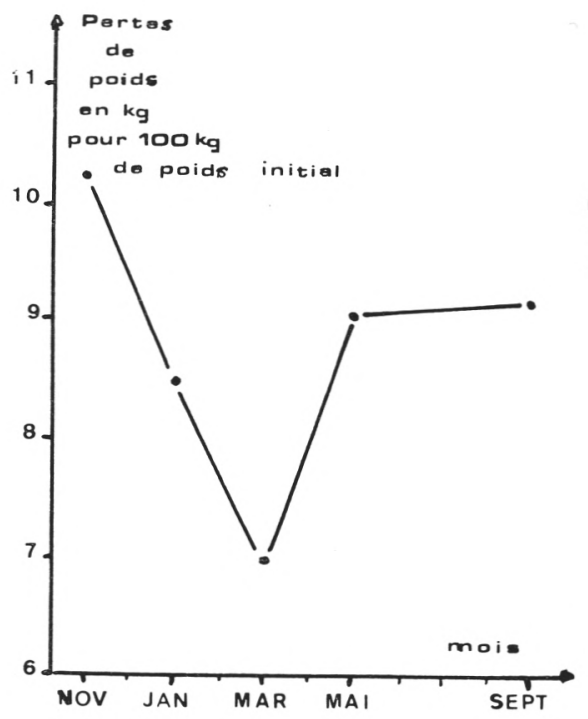

GRAPHIQUE no 4

Evolution de la perte de poids

des fromages an fanction de la

saison et de la zone góographique

Fromages INRA I

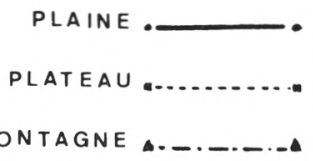

MONTAGNE

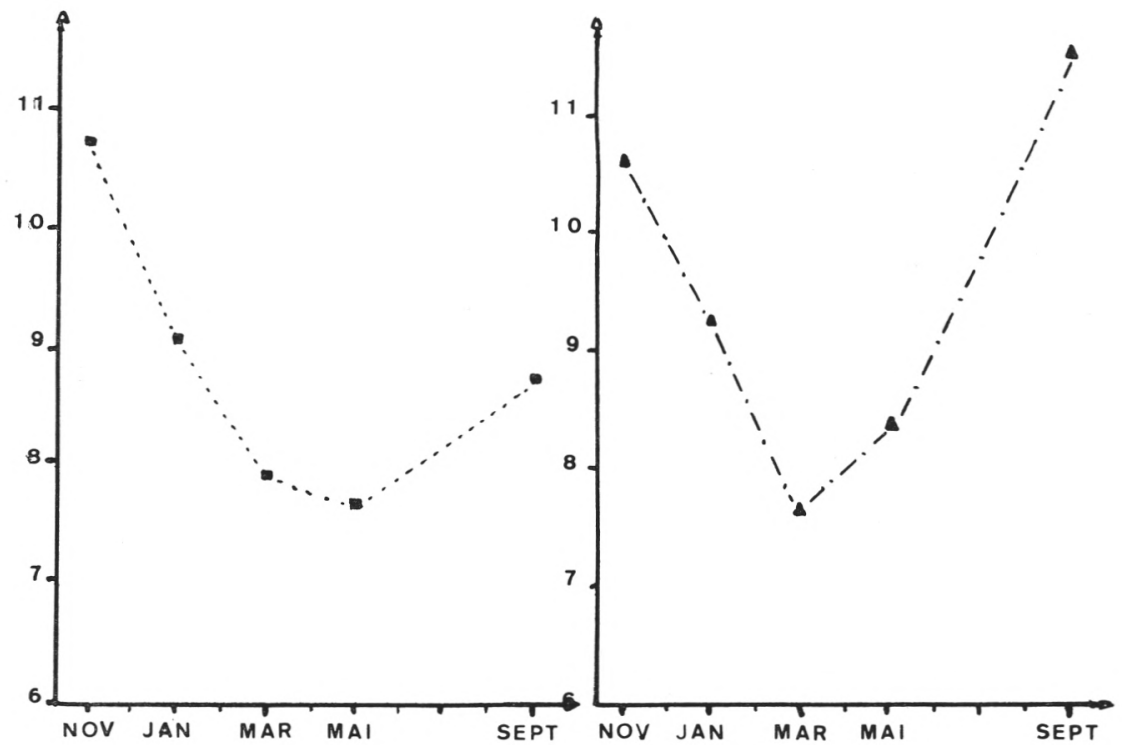

graphique 4 
ont une perte de poids proportionnellement plus importante que les plus petits serait à vérifier sur un échantillon plus grand puisqu'en effet, les corrélations calculées sont $r=-0,22$ pour les fromages fabriqués à la fruitière d'origine et $r=-0,46$ (limite de la signification à $5 \mathrm{p} .100$ ) pour les fromages INRA I.

b) Extrait sec à $20 \mathrm{~h}$

Il est généralement admis qu'un fromage relativement humide a tendance à perdre d'avantage de poids qu'un fromage plus sec. Nous n'avons cependant pas pu mettre en évidence de corrélations significatives entre ces deux variables $(r=0,007)$ pour les fromages « fruitières I " et $(r=0,26)$ pour les fromages INRA I.

\section{c) Taux azoté du lait de fabrication}

Il existe une bonne corrélation $(\mathrm{r}=+0,736)$ entre la perte de poids des fromages (INRA I) au cours de l'affinage et la teneur en matière azotée du lait de fabrication en chaudière.

\section{Analyse sensorielle}

L'exploitation des tests de dégustation effectués sur les fromages INRA II, fruitière I et fruitière II de chacune des trois fruitières et sur cinq périodes de l'année (effectif 45 fromages) nous permet de comparer les fromages entre eux.

Les trois critères retenus, ouverture, texture et goût sont jugés par des notes de 1 à 9 ( 9 étant la qualité extra).

\section{a) Ouverture des fromages}

L'ouverture des fromages est notée à 6 mois. Les 45 fromages ont une ouverture moyenne de 5,6 (c'est-à-dire une ouverture jugée plutôt belle), 29 p. 100 (13/45) des fromages ont une ouverture jugée belle à très belle et 15,5 p. 100 (7/45) vilaine à très vilaine (1 à 4).

De l'étude statistique il ressort différents points :

Le critère " ouverture " permet de mettre en évidence des différences significatives $(\mathrm{P}<0,01)$ entre les fromages des trois fruitières. Bien qu'il y ait des différences notables entre dégustateurs pour les mois de novembre, janvier et mars.

Le classement des fromages par fruitière fait ressortir des différences marquées dues notamment à l'origine du lait et à la technologie. C'est ainsi que les fromages de $\mathrm{C}$ sont les mieux notés avec en général une belle ouverture, par contre ceux de A sont les moins bien jugés en novembre, janvier et mars.

En se référant aux fromages, fabriqués à la fromagerie expérimentale INRA avec le lait des trois fruitières, les différences sont beaucoup moins marquées (sauf en janvier), ceux-ci étant assez homogènes dans l'ensemble. Cependant les fromages fabriqués avec le lait de $\mathrm{C}$ sont les mieux notés et ceux de la fromagerie $\mathrm{B}$ les plus mal jugés 
(sauf en septembre). Par conséquent, l'origine du lait semble avoir une influence sur l'ouverture des fromages. Mais si l'on compare le classement général de tous les fromages dégustés avec le seul classement des fromages INRA, on s'aperçoit que les fromages de A, qui sont les plus mal placés dans l'ensemble des fromages, ne le sont plus dans le cas des fromages INRA et inversement pour les fromages de B. On peut penser que la technologie appliquée à A n'est pas adaptée au lait.

La comparaison entre les fromages INRA et les fromages F1 et F2 de la fruitière d'origine montre qu'au niveau de la fruitière $A$, les fromages INRA ont une ouverture plus régulière et sont mieux notés pour les séries de novembre, janvier et mars, que les fromages fabriqués à la fruitière d'origine (souvent chargés, éraillés et aux yeux non francs). Ceci confirme les résultats précédents et montre que A n'est pas exempt de problèmes technologiques.

Quant à $\mathrm{B}$, on observe le phénomène inverse. Les fromages INRA (janvier, mars, mai) ; (insuffisamment ouverts et avec défauts sous croûte), sont bien moins notés que les fromages de la fruitière d'origine. La technologie du fromager B semble mieux adaptée au lait non transporté que celle appliquée à la fromagerie expérimentale pour un tel lait transporté. En ce qui concerne le lait de C, les fromages fabriqués à la fruitière d'origine ou à l'INRA ont en général une ouverture assez homogène (bien que parfois apparaissent quelques becs de lainure). La technologie, qu'elle soit de $\mathrm{C}$ ou de la fromagerie expérimentale paraît adaptée au lait sans ou avec transport.

\section{b) Texture des fromages}

L'étude statistique des notes accordées aux 45 fromages pour leur texture à 3,5 et 6 mois n'a relevé aucune différence significative. Ces derniers ont une note moyenne de texture de 6,9 (bonne), dont 11 p. $100(5 / 45)$ ont une très belle texture, par contre aucun des fromages n'a été trouvé mauvais.

Le critère " texture " ne permet pas de mettre en évidence des différences entre fromages et entre zones géographiques. La texture est cependant assez bien jugée sur l'ensemble de la série.

\section{c) Goût des fromages}

Le goût des fromages est une notion subjective, souvent difficile à tester, du fait d'une différence d'appréciation pouvant exister d'un dégustateur à l'autre. A la suite de l'étude statistique sur les 45 fromages, seules les séries de mars à 3 mois et de mai à 5 mois, relèvent des différences significatives entre fromages, qui d'ailleurs ne se retrouvent pas par la suite. Il faut attendre 6 mois pour voir apparaître quelques différences. Ces fromages ont en moyenne un goût 
plutôt bon, de 6,1, puisque 15,5 p. 100 (7/45) des fromages sont jugés bons à très bons et seulement 4,4 p. 100 (2/45) ont un mauvais goût :

- la notation globale des fromages est assez homogène, seules des différences significatives entre eux apparaissent au mois de janvier ;

- le classement par fruitière montre, que pour les séries de novembre et septembre, les fromages de C ont un "meilleur goût " (francs et fruités), que ceux de A (plutôt fades). L'influence de l'origine du lait et de la technologie est à peine sensible ;

- quant aux fromages INRA, eux aussi sont assez homogènes et jugés bons dans l'ensemble sauf pour la fabrication de mai et septembre où certains sont trouvés amers. L'origine géographique du lait aurait peu d'influence sur le goût des fromages. Par contre pour la série de septembre, on pourrait mettre en évidence l'influence de la technologie au niveau de la fruitière A.

A l'intérieur d'une même fruitière, les différences entre fromages INRA et fromages de la fruitière d'origine sont assez peu sensibles.

Au niveau de la fruitière de A, le seul résultat significatif montre que le fromage F2 (qui a été trouvé très piquant) est mal noté par rapport aux deux autres fromages, dans la série de janvier. Sur l'ensemble de la fruitière les fromages INRA sont en général mieux notés que ceux de la fruitière d'origine qui sont souvent peu francs, amers ou piquants. Ceci serait en partie dû, là encore, au facteur " technologie".

\section{DISCUSSION - CONCLUSION}

Les résultats présentés nous permettent de soumettre quelques conclusions qu'il faut cependant recueillir avec prudence, compte tenu des conditions expérimentales. En effet, une fruitière par zone, ne peut en aucun cas être représentative de la zone et, par voie de conséquence, les résultats émanant de cette fromagerie ne peuvent être extrapolables à la zone.

La zone géographique ne semble pas être un facteur de variation déterminant de la composition chimique des laits ; ceci confirme nos précédentes observations mais semble en contradiction avec les travaux de Auriol et Mocquot [1] une amélioration des méthodes d'élevage pourrait en être la raison.

Pour les fromages à 6 mois, les conditions géographiques indépendamment des conditions technologiques, semblent influer sur leur composition chimique mais surtout sur leur qualité. Aussi la fruitière $C$, fruitière de montagne, fournit en moyenne des fromages de meilleure qualité que ceux de A (plaine) et B (plateau). La qualité 
bactériologique du lait en est-elle une des causes ? On peut se le demander.

La saison constitue un important facteur de variations aussi bien de la composition chimique des laits et des fromages que de la qualité de ces derniers.

Les teneurs en matières grasses et azotées du lait sont maximums en automne et minimums en janvier-mars ; ceci est en accord avec les observations de S. Kuzdzal-Savoie et W. Kuzdzal [4], Decaen et Journet [3] et de Decaen et Ghadaki [2], bien qu'au mois de mai on n'observe pas une remontée très nette du taux butyreux et du taux azoté.

Pour les fromages, les mois de mai et de novembre constituent les deux extrêmes. L'extrait sec, la mesure des matières azotées au noir amido sont minimums en novembre et maximums en mai ; il en est de même pour le pourcentage molaire d'acides gras longs. Ces 2 mois constituent le début et la fin de la saison de pâturage, aussi l'alimentation constitue certainement un facteur de variation non négligeable qui mériterait d'être analysé dans une étude ultérieure. Les pertes de poids (observées à 4 mois d'affinage) sont les plus faibles en mars et les plus fortes en novembre.

Enfin la conclusion essentielle de ce travail est que les conditions technologiques sont primordiales pour la qualité du Comté. En effet l'hétérogénéité de qualité entre les trois fruitières, quelle que soit la saison, est fortement réduite lorsque les fromages sont fabriqués à Aresches selon un procédé unique. Ceci met en évidence l'aspect plus important de la technologie fromagère par rapport à l'origine géographique du lait. Par conséquent, il nous apparaît souhaitable de continuer ce travail sur un nombre plus important de fruitières de montagne et de plaine et par une analyse précise des techniques de fabrication, afin de répondre aux questions que se posent les producteurs de la région au sujet de la qualité de leurs fromages.

\section{Remerciements}

Nous remercions tous les professionnels de la région qui nous ont aidés à réaliser cette étude et notamment les Etablissements Grillot de Poligny, les Présidents et fromagers des Fruitières de Buffard, Goux-sous-Landet et Lièvremont-village et l'Ecole Nationale d'Industrie laitière de Poligny.

\section{Rés u mé}

Les variations saisonnière et géographique de la composition du lait et du fromage de Comté sont étudiées à partir d'échantillons provenant de trois fromageries réparties sur trois zones (plaine $400 \mathrm{~m}$, plateau 400 à $800 \mathrm{~m}$ et montagne $>800 \mathrm{ml}$ ) et prélevés cinq fois dans l'année, novembre, janvier, mars, mai et septembre. 
La composition chimique (taux de matières azotées et de matières grasses, composition en acides gras des lipides), des laits et des fromages à 6 mois d'affinage varie beaucoup plus en fonction de la saison que de la zone géographique. La teneur en matières azotées des laits, maximum en septembre $(33,4 \mathrm{~g} / \mathrm{kg})$ et minimum en mars $(30,9 \mathrm{~g} / \mathrm{kg})$, varie seulement entre $32 \mathrm{~g} / \mathrm{kg}$ et $32,4 \mathrm{~g} / \mathrm{kg}$ en fonction de la zone géographique.

Les lipides du lait de la période (mai-septembre) sont par rapport à ceux du lait de la période (novembre-janvier et mars) plus riches en acides gras courts $(24,4$ contre 21,8 p. 100$)$ plus pauvres en acides gras moyens (36 contre 46,8 p. 100) et plus riches en acides gras longs (38,3 contre 31,2 p. 100).

L'évolution de la composition chimique et de la perte de poids est analysée au cours des 6 mois d'affinage par des prélèvements à 20 h, 3, 4, 5, et 6 mois.

L'extrait sec minimum à 20 h (63 p. 100) augmente régulièrement jusqu'au $5^{\mathrm{e}}$ mois d'affinage (65 p. 100).

La perte de poids observée entre le stade $20 \mathrm{~h}$ et 4 mois d'affinage est représentative de la perte globale, elle oscille entre 7 et 10 p. $100 \mathrm{du}$ poids du fromage à $20 \mathrm{~h}$.

L'ouverture, la texture et le goût sont appréciés au cours de tests de dégustation. Seule l'ouverture permet de mettre en évidence des différences entre fromages. En définitive, les conditions technologiques de fabrication apparaissent primordiales pour la qualité du Comté.

\section{S u m m a ry}

\section{GEOGRAPHICAL AND SEASONAL VARIATIONS OF COMTE CHEESE QUALITY}

Seasonal and regional differences in gross composition of manufacturing milk and Comte cheese (gruyere Swiss-type cheese) were studied in 3 cheese factories located in the Jura mountains at different elevation: plain $(400 \mathrm{~m})$, plateau $(400-800 \mathrm{~m})$ and mountain $(>800 \mathrm{~m})$ and sampled in Nov., Jan., May and Sept.

Chemical composition (protein, fat and fatty acids) of milk and of 6 month old cheeses vary much more according to the season that to the geographical origin. Milk protein content is maximum in September $(33,4 \mathrm{~g} / \mathrm{kg})$ and minimum in March $(30,9 \mathrm{~g} / \mathrm{kg})$, the average range of variation between areas is low (from 32 to $32,4 \mathrm{~g} / \mathrm{kg}$ ).

Compared to the period of Nov.-Jan.-March, fatty acids composition of milk samples from May-September are richer in short chain and long chain (38.3 vs, 31.2 p. 100 and 24.4 vs, 21.8 p. 100) and lower in medium chain (36 vs, 46.8 p. 100). 
Percentage of dry matter of cheese is minimum at $20 \mathrm{~h}(63 \mathrm{p} .100)$ and increases regularly till the 5 th month of ripening (65 p. 100).

Weight lost of cheeses is maximum at the 4th month and vary between 7 and 10 p. 100 of the weight at $20 \mathrm{~h}$.

Holes, body, taste and flavour of cheeses were tested. Only the development of eyes was found statistically different between cheeses. Finaly, processing conditions appears to be of prime importance for the quality of Comte cheese.

Reçu pour publication en août 1980.

\section{Bibliographie}

[1] Auriol (P.) et Mocouot (C.) (1957). - Variations saisonnières de la composition des laits d'étable et de fromagerie dans la région de Gruyère. Ann. Zootechn., 6, 95-120.

[2] Decaen (C.) et GhadaKi (M. B.) (1970). - Variations de la sécrétion des acides gras des matières grasses du lait de vache à la mise à l'herbe et au cours des 6 premières semaines d'exploitations du fourrage vert. Ann. Zootech., 19, 399-411.

[3] Decaen (C.) et Journet (M.) (1966). - Influence saisonnière sur la production et la composition du lait. Ann. Zootech., 15, 259-277.

[4] Kuzdzal-Savore (S.) et Kuzdzal (W.) (1961). - Influence de la mise à l'herbe des vaches laitières sur les indices de la matière grasse du beurre sur les teneurs en différents acides gras poly-insaturés. Ann. Biol. Anim. . Bioch., Biophys., 1, 47-69.

[5] Masson (C.), Decaen (C.) et Rousseaux (P.) (1978). - Variations géographique et saisonnière de la composition du lait destiné à la fabrication de Gruyère de Comté. Le Lait, 575-576, 261-273.

[6] Maurer (L.) et Stock (H.) (1978). - Teneur en chlorure de sodium des fromages Emmental autrichiens et son rapport avec les défauts du fromage. Brèves communications. 20 Congrès Intern. de Laiterie, Paris. 